\title{
Molecular and Clinical Characterization of a Claudin-Low Subtype of Gastric Cancer
}

Tomohiro F. Nishijima

Jordan Kardos

Shengjie Chai

Christof C. Smith

Dante S. Bortone

Sara R. Selitsky

Joel S. Parker

Hanna K. Sanoff

Michael S. Lee

Benjamin G. Vincent

Author affiliations appear at the end of this article.

Supported by the

University Cancer

Research Fund of the

Lineberger

Comprehensive Cancer

Center at the University of

North Carolina at Chapel

Hill and the University of

North Carolina at Chapel

Hill Oncology Clinical

Translational Research

Training Program

(5K12CA120780).

Presented at the ASCO

2017 Gastrointestinal

Cancers Symposium, San

Francisco, CA, January 19-

21, 2017

Corresponding author:

Benjamin G. Vincent, MD

Lineberger

Comprehensive Cancer

Center, University of

North Carolina at Chapel Hill, 125 Mason Farm Rd,

Chapel Hill, NC 27599;

e-mail: benjamin_vincent@

med.unc.edu.

Purpose Claudin-low molecular subtypes have been identified in breast and bladder cancers and are characterized by low expression of claudins, enrichment for epithelial-to-mesenchymal transition (EMT), and tumor-initiating cell (TIC) features. We evaluated whether the claudin-low subtype also exists in gastric cancer.

Materials and Methods Four hundred fifteen tumors from The Cancer Genome Atlas (TCGA) gastric cancer mRNA data set were clustered on the claudin, EMT, and TIC gene sets to identify claudin-low tumors. We derived a 24 -gene predictor that classifies gastric cancer into claudinlow and non-claudin-low subtypes. This predictor was validated with the Asian Cancer Research Group (ACRG) data set. We characterized molecular and clinical features of claudin-low tumors.

Results We identified 46 tumors that had consensus enrichment for claudin-low features in TCGA data set. Claudin-low tumors were most commonly diffuse histologic type (82\%) and originally classified as TCGA genomically stable (GS) subtype (78\%). Compared with GS subtype, claudin-low subtype had significant activation in Rho family of GTPases signaling, which appears to play a key role in its EMT and TIC properties. In the ACRG data set, 28 of 300 samples were classified as claudinlow tumors by the 24-gene predictor and were phenotypically similar to the initially derived claudinlow tumors. Clinically, claudin-low subtype had the worst overall survival. Of note, the hazard ratios that compared claudin-low versus GS subtype were 2.10 (95\% CI, 1.07 to 4.11 ) in TCGA and 2.32 (95\% CI, 1.18 to 4.55$)$ in the ACRG cohorts, with adjustment for age and pathologic stage.

Conclusion We identified a gastric claudin-low subtype that carries a poor prognosis likely related to therapeutic resistance as a result of its EMT and TIC phenotypes.

Precis Oncol 00. @ 2017 by American Society of Clinical Oncology

\section{INTRODUCTION}

Claudin-low tumors have been identified as a molecular subtype originally in breast cancer and more recently in bladder cancer on the basis of gene expression profiling. ${ }^{1,2}$ These distinct tumors are characterized by the low expression of tight-junction claudins, enrichment for epithelial-to-mesenchymal transition (EMT) markers, and tumor-initiating cell (TIC) features. ${ }^{1,2}$ Clinically, claudin-low tumors, which lack luminal differentiation marker expression, are associated with poor prognosis compared with luminal tumors. ${ }^{1,3}$

Similar to breast, bladder, and other types of cancer, gastric cancer is a heterogeneous disease and arises from multiple genetic and epigenetic aberrations. To better understand the biology that drives gastric cancer, molecular evaluation of gastric cancer has been performed and has led to the identification of key dysregulated pathways and the development of molecular classifications. ${ }^{4}$ Gene expression-based classifiers were reported by the Asian Cancer Research Group (ACRG) ${ }^{5}$ and by Lei et al. ${ }^{6}$ The ACRG divided gastric cancer tumors into four subtypes (microsatellite unstable [MSI], EMT, and tumor protein 53 active and inactive), and Lei et al classified gastric cancer tumors into three subtypes (proliferative, metabolic, and mesenchymal).

On the basis of an integrative analysis of molecular profiling data, The Cancer Genome Atlas (TCGA) proposed the following four molecular subtypes of gastric cancer tumors: Epstein-Barr virus (EBV) positive, MSI, chromosomally unstable (CIN), and genomically stable (GS). ${ }^{7}$ This classification system first categorizes tumors by 
Fig 1. Identification of a claudin-low subtype in gastric cancer. (A) Unsupervised clustering of The Cancer Genome Atlas (TCGA) gastric cancer samples. Samples were clustered on the basis of expression of tight-junction claudins, a bidirectional epithelial-to-mesenchymal transition (EMT) signature, and a tumorinitiating cell (TIC) signature.
EBV-positivity and then by MSI-high status; the remaining tumors are distinguished by degree of aneuploidy. GS subtype is less well characterized by this decision tree because it is distinguished from CIN just on the basis of the absence of extensive somatic copy number aberrations. However, GS subtype was found to have some notable features, including enrichment of Lauren diffuse histologic type and alterations in the genes associated with cell adhesion and motility, such as $C D H 1$ and $R H O A$ mutations and CLDN18-ARHGAP fusions. In addition, comparisons between TCGA and ACRG classification systems revealed that TCGA GS was enriched in ACRG EMT. ${ }^{5}$ These findings suggest that some GS tumors share molecular characteristics with claudin-low tumors. Therefore, we evaluated whether a claudin-low subtype also exists in gastric cancer.

\section{MATERIALS AND METHODS}

\section{Data Sets}

TCGA stomach adenocarcinoma (STAD) RNA expression data set was downloaded from the Broad Institute Firehose Pipeline ${ }^{8}$ on February 17, 2016. Expression values were $\log ^{2}$-transformed, and genes with $<80 \%$ expression across all samples

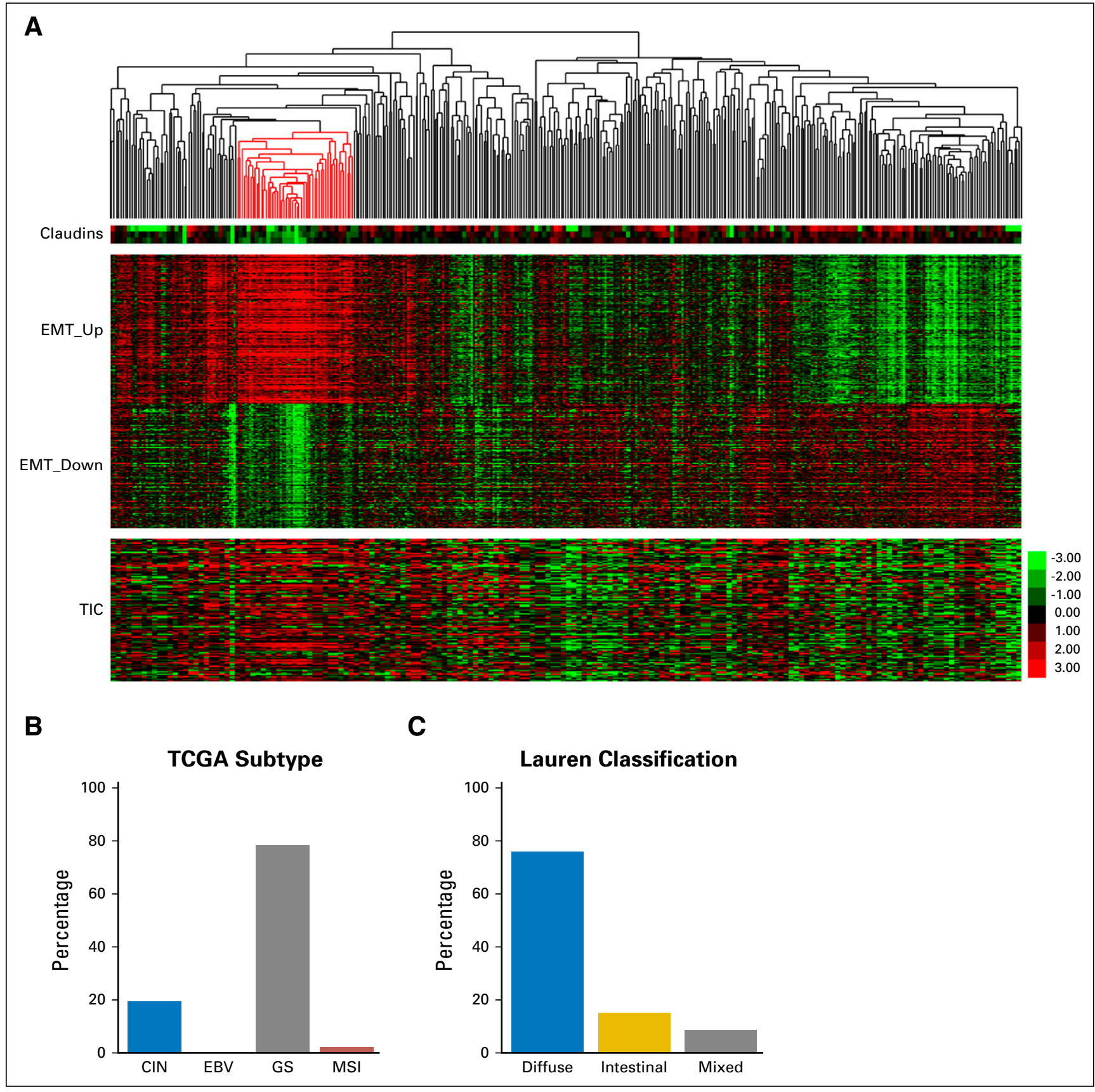


The tumors identified as claudin-low are highlighted in red on the dendogram (n =415). Bar graphs show the classification of 46 claudinlow tumors by (B) TCGA subtype and by (C) Lauren histologic type. CIN, chromosomally unstable; EBV, Epstein-Barr virus; GS, genomically stable; MSI, microsatellite unstable. were filtered out. Missing values were imputed using the k-nearest neighbor imputation method, and gene expression values were median centered across each gene.

\section{Gene Expression Signatures}

Tight-junction claudin, EMT, and TIC gene signatures were used in the classification of a claudin-low subtype. The set of claudins used was identified by Herschkowitz et al. ${ }^{9}$ The bidirectional Pan-Cancer EMT signature was derived by Tan et al. ${ }^{10}$ The gastric cancer-specific TIC signature was derived from the publicly available Gene Expression Omnibus gene expression data set (GSE53276). Genes were filtered for a significant difference between the control and tumorigenic cell line 60As6 using the significance analysis of microarrays with a false discovery rate $<0.01$ and for their presence in TCGA STAD data set. We identified a panel of 119 genes that are upregulated in gastric cancer TICs (Data Supplement). Proliferation and immune gene signatures were derived by Hippo et $\mathrm{al}^{11}$ and Iglesia et al, ${ }^{12}$ respectively.

\section{Identification of a Claudin-Low Subtype}

Data were clustered on the claudin, EMT, and TIC gene sets using average linkage clustering with a centered correlation similarity metric on the Cluster 3.0 platform (Stanford University, Stanford, CA). A conservative node with lowclaudin, high EMT-UP, low EMT-DOWN, and high TIC gene sets was selected. SigClust2 R software (Statistical Significance of Clustering 2) was run on the node to perform a Gaussian distribution analysis, which expands to the entire gene set for each increasing node. Tumors identified by SigClust 2 were called claudin-low subtype regardless of the original TCGA subtype call. Breast cancer claudin predictions were also made on the 415-sample TCGA STAD data set using the distance-weighted discrimination claudin classifier, which is based on the 807-gene signature provided by Prat et al. ${ }^{1}$ We applied prediction analysis of microarrays to the 415-sample TCGA STAD data set to derive a gastric claudin-low or other subtype classifier. A threshold of 10.9 was selected, which gives a 24-gene predictor with an overall error rate of 0.055 . An independent set of 300 gastric cancer samples from the ACRG data set (Gene Expression Omnibus GSE62254) served as a validation set. The data set was mean collapsed onto genes and then median centered. The Data Supplement contains additional details on methods.

\section{RESULTS}

Identification of a Claudin-Low Subtype in Gastric Cancer

We performed unsupervised hierarchical clustering on 415 gastric cancer samples from TCGA data set using gene signatures representative of biologic characteristics known to define the claudin-low subtype of breast and bladder cancers. ${ }^{1,2}$ This unsupervised hierarchical clustering with these gene signatures revealed a distinct cluster that had characteristics of claudin-low tumors (Fig 1A, highlighted in red). To ensure that the set of tumors within the presumed claudin-low cluster were homogeneous and distinct from adjacent clusters of tumors, we performed a Gaussian distribution analysis (Data Supplement). This method identified a conserved node of 46 tumors $(11.1 \%)$ that had consensus enrichment for claudin-low features, and these tumors, therefore, were defined as claudin-low subtype. Classification of the non-claudin-low tumors $(\mathrm{n}=369)$ was as follows: EBV, 31 tumors; MSI, 79 tumors; CIN, 218 tumors; and GS, 41 tumors. As we hypothesized, claudin-low tumors were primarily found in the diffuse histologic type and TCGA GS subtype (Figs 1B and 1C). Because the claudin-low tumors were identified originally in breast cancer, we applied the previously defined breast cancer-specific claudin-low classifier to TCGA gastric cancer samples and found a significant enrichment $(P<.001$, Fisher's exact test $)$ of the breast cancer-defined claudin-low tumors within the gastric claudin-low cluster (Data Supplement). This finding further supports the notion that claudin-low tumors exhibit features of previously defined claudin-low tumors.

\section{Gene Expression Signature Analysis}

Relative to TIC and stem-cell features, breast cancer claudin-low tumors express low levels of proliferation genes and are likely slowercycling tumors. ${ }^{1,3} \mathrm{We}$ examined proliferation gene expression of gastric cancer by subtype using a previously reported gastric cancer-specific proliferation signature. ${ }^{11}$ Expression of the proliferation-associated genes in the claudinlow subtype was lowest among all the subtypes (Figs 2A and 2B). Despite the apparent similarity to GS subtype, the claudin-low subtype expressed significantly lower levels of proliferation genes than GS subtype $(P<.001$, Bonferronicorrected pairwise $t$ test). This finding supports that the claudin-low subtype of gastric cancer is a unique subtype with distinct biologic properties. 
Fig 2. Proliferation gene expression by gastric cancer subtypes. (A) Heat map of supervised clustering of gastric cancer subtypes across previously identified proliferationassociated genes $(n=415)$. (B) Box plot of proliferation gene signature $z$ scores across gastric cancer subtypes $(n=415)$. Significance was determined by one-way analysis of variance (ANOVA). CIN, chromosomally unstable; EBV, Epstein-Barr virus; GS, genomically stable; MSI, microsatellite unstable.
Another notable feature of the claudin-low subtype of breast and bladder cancers is high expression of immune cell genes, including $\mathrm{T}$ and $\mathrm{B}$ cells. ${ }^{1,2}$ To characterize the immune response in gastric claudin-low tumors, we evaluated gene signatures associated with immune cells by molecular subtype using previously defined signatures that correspond to tumor-infiltrating immune cells. ${ }^{12}$ Heat maps of immune signature expression across the 415 samples in order by subtype showed generally high expression in EBV, claudin-low, and GS (Fig 3A). On the basis of the $z$ scores of the gene signature, expression of all immune signatures other than the B-cell signature was highest in the EBV subtype (Data Supplement).

To assess the level of active immunosuppression, we examined the expression of a previously defined panel of immune checkpoint molecules (immunosuppression signature $)^{2}$ and found that it was also highest in the EBV subtype (Fig 3B; Data Supplement). This observation is in line with the previous report by Strong et al. ${ }^{13}$ wherein EBVpositive gastric carcinoma samples expressed high levels of the cytotoxic T-cell and natural killer cell inhibitor indoleamine-2,3-dioxygenase 1 , which leads to the induction of immune tolerance to tumors despite the elevated immune cell infiltration. Similar to the previous analysis of bladder cancer, a clear correlation existed between the immune signatures and the immunosuppression signature across all gastric cancer subtypes ${ }^{2}$ (Data Supplement).

The prognostic impact of tumor-immune infiltrates, especially tumor-infiltrating lymphocytes (TILs), has been reported in multiple tumor types. ${ }^{14,15}$ For instance, the presence of $\mathrm{CD}^{+}$ TILs was associated with favorable survival outcomes. ${ }^{16-18}$ Some studies evaluated the prognostic relevance of TILs in gastric cancer, but the results were not consistent across them. ${ }^{19-21} \mathrm{We}$ performed Cox proportional hazards regression modeling for each immune gene signature, including immunosuppression signature, across all tumors and within each subtype. None of the signatures were prognostic in TCGA gastric cancer samples (data not shown).

We explored potential mechanisms of immune response by examining predicted neoantigen burden and levels of cytokines and chemokines among subtypes. Neoantigens are altered peptides derived from tumor-intrinsic mutant proteins that can elicit antitumor T-cell responses. We predicted neoantigens using a previously described bioinformatics pipeline that takes as input exome
A

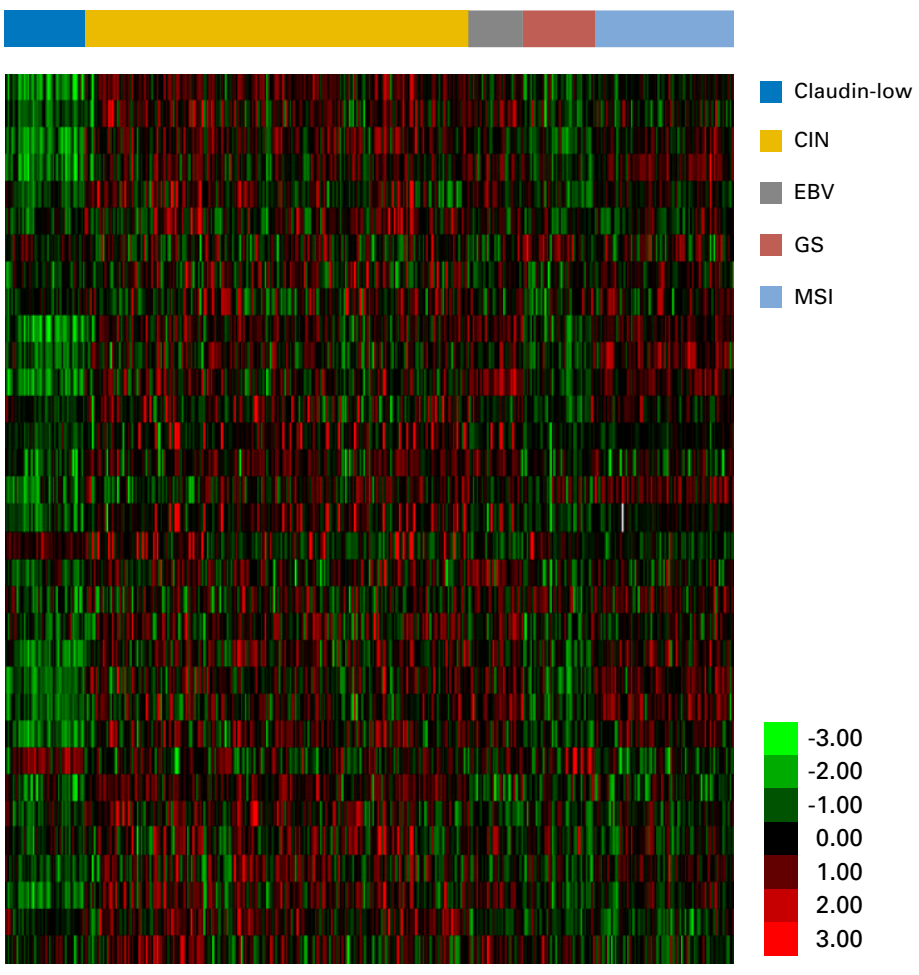

B

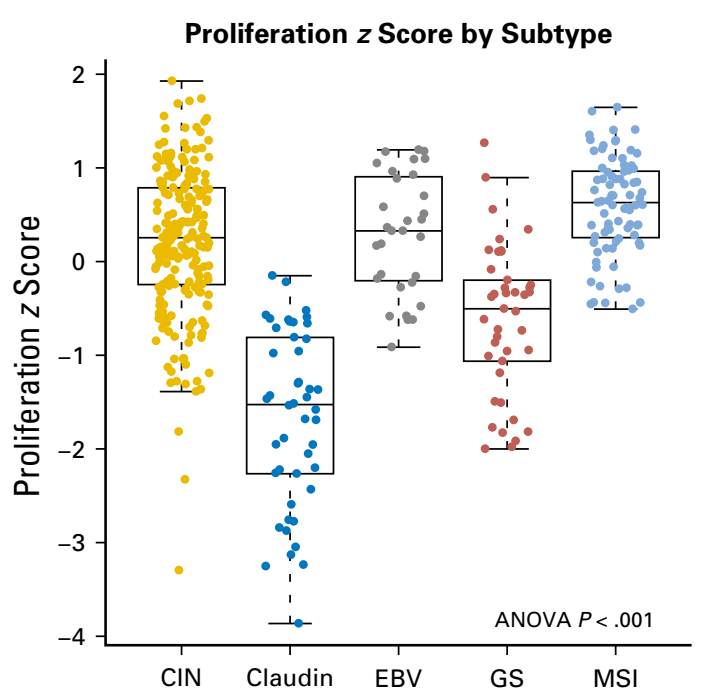




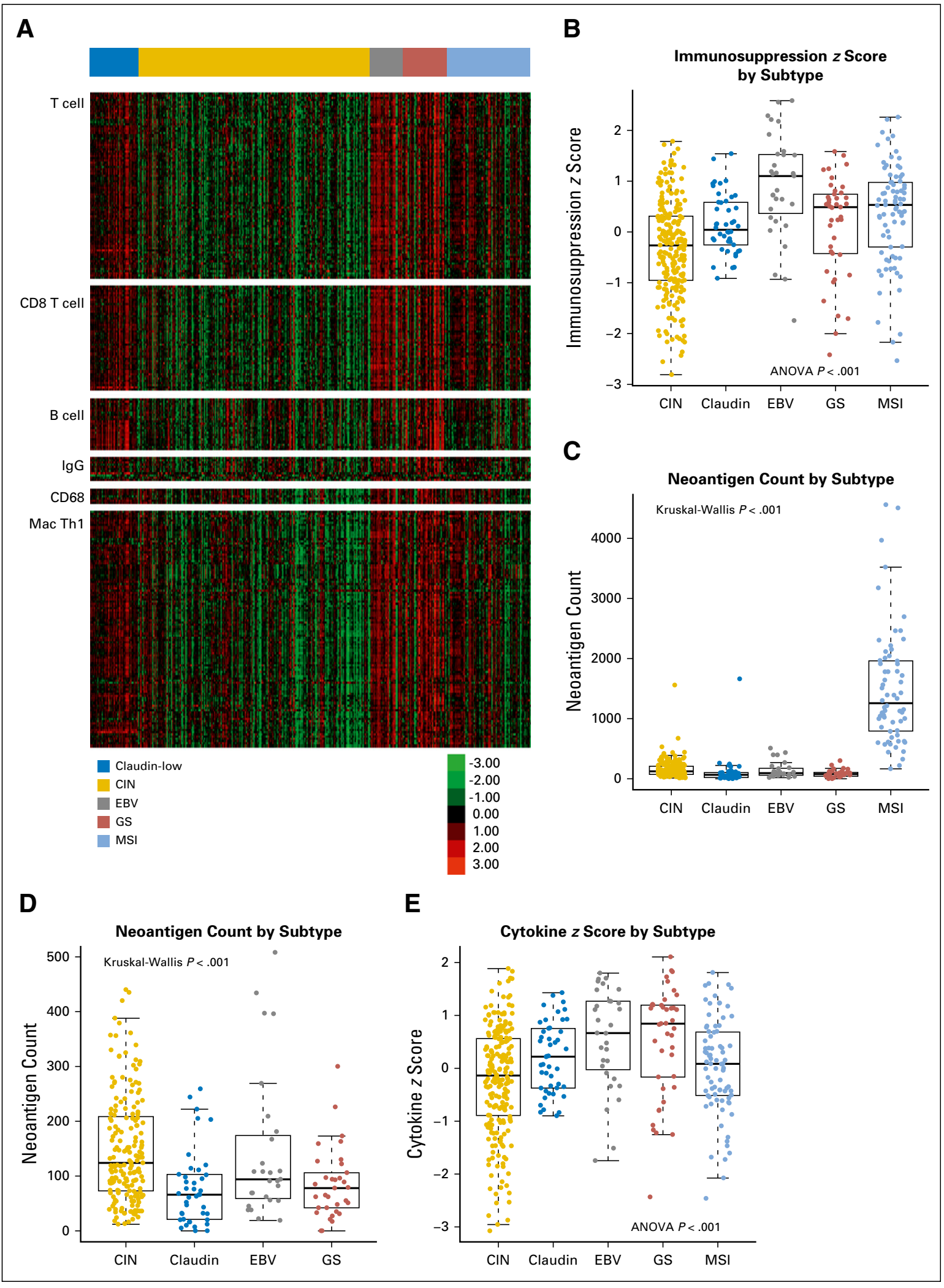

Fig 3. Immune characterization of gastric cancer subtypes. (A) Heat maps of supervised clustering of gastric cancer sequencing and mRNA sequencing data. ${ }^{2}$ A strong correlation was found between predicted neoantigen burden and the number of somatic mutations (Pearson $r=0.895 ; P<.001$; Data
Supplement). Not surprisingly, MSI subtype had a significantly higher predicted neoantigen burden than the other subtypes (Fig 3C). A level of predicted neoantigen could not explain the 
subtypes across previously identified immune signatures $(\mathrm{n}=415)$. (B) Box plot of immune suppression gene signature $z$ scores across gastric cancer subtypes $(n=415)$.

Significance was determined by one-way analysis of variance (ANOVA). (C) and (D) Box plots show the number of predicted neoantigens with a half-maximal inhibitory concentration $<150 \mathrm{~nm}$ by tumor molecular subtype. Significance was determined by KruskalWallis exact test $(\mathrm{n}=357)$. (E) Box plot of cytokine and chemokine signature $z$ scores across gastric cancer subtypes $(\mathrm{n}=415)$.

Significance was

determined by one-way ANOVA. CIN, chromosomally unstable; EBV, Epstein-Barr virus; GS, genomically stable; IgG, immunoglobulin G; Mac Th1, macrophageassociated Thelper 1; MSI, microsatellite unstable. difference in immune infiltration among subtypes because the predicted neoantigen burdens of EBV, claudin-low, and GS subtypes were lower than that of CIN subtype and relatively similar to one another (Fig 3D).

We next examined the relative expression of a previously defined panel of cytokines and chemokines by subtype ${ }^{2}$ and observed a strong correlation between this expression signature and multiple immune signatures, including immunosuppression signature (Data Supplement). EBV and GS subtypes had a high level of cytokine and chemokine expression (Fig 3E). The increased production of proinflammatory cytokines and chemokines may contribute to immune infiltrate in these subtypes. Overall, claudin-low tumors appeared to have a high level of immune infiltration but lacked active immunosuppression within the tumor microenvironment.

\section{Pathway Analysis}

Given that claudin-low tumors were primarily found in GS subtype, we performed ingenuity pathway analysis (IPA) and gene set enrichment analysis to understand the gene expression patterns that differentiate claudin-low tumors from non-claudin-low GS tumors. IPA revealed that claudin-low subtype had significant activation in Rho family of GTPases signaling and significant inactivation in Rho guanine nucleotide dissociation inhibitor (GDI) signaling compared with GS subtype (Fig 4). RhoA (a member of the Rho family of GTPases) signaling has been reported to promote cancer stem-cell-like phenotype in diffuse-type gastric cancer. ${ }^{22}$ In addition, claudinlow subtype had higher levels of actin cytoskeleton and integrin signaling relative to signaling levels in GS subtype. Furthermore, gene set enrichment analysis demonstrated enrichment of cell motility and adhesion-associated pathways in claudinlow subtype (Data Supplement). These observations are in keeping with the TIC and EMT phenotypes, which are defining characteristics of claudin-low tumors.

\section{Mutation and Copy Number Alteration Analysis}

We next examined somatic mutations and copy number alterations using TCGA data set to compare genomic events of claudin-low subtype with those of non-claudin-low GS subtype (Data Supplement). TCGA network reported $R H O A$ and CDH1 mutations, and CLDN18-ARHGAP fusions were enriched in GS subtype. ${ }^{7}$ We did not observe significant differences in the frequency of these genomic events between the two subtypes (Data Supplement). However, the claudinlow subtype had a higher prevalence of CD44, GATA4, and GATA6 amplifications than the GS subtype. $C D 44$ is a gastric cancer stem-cell marker and involved in cell adhesion and migration. ${ }^{23}$ GATA4 and GATA6 are transcription factors involved in gastric cancer tumorigenesis as lineagesurvival oncogenes. ${ }^{24}$ Amplification of these genes and their subsequent effects on signal transduction and transcription may be partially responsible for differences in gene expression between the subtypes.

\section{A 24-Gene Classifier Accurately Predicts Claudin-Low Tumors}

To define a minimal set of genes that could accurately classify claudin-low gastric cancer, we applied prediction analysis of microarrays to TCGA STAD data set and derived a 24-gene predictor (Data Supplement), which accurately classifies gastric cancer into claudin-low and nonclaudin-low subtypes, with a training error rate of 0.15 and 0.04 , respectively. To validate this predictor, we made claudin-low subtype calls on an independent set of 300 gastric cancer samples from the ACRG data set. We also made TCGA subtype calls (EBV, MSI, CIN, or GS) on the 300 tumors by following TCGA classification. Tumors identified by the claudin-low predictor were called claudin-low subtype regardless of TCGA subtype call. The claudin-low predictor identified 28 claudin-low tumors (9.3\%) in the ACRG data set. Non-claudin-low tumors $(\mathrm{n}=272)$ were classified into the following subtypes: EBV, 17 tumors; MSI, 63 tumors; CIN, 162 tumors; and GS, 30 tumors. We found that claudin-low subtype in the ACRG data set was phenotypically similar to the initially derived claudin-low subtype in the discovery TCGA data set as measured by expression of the claudin, EMT, and TIC gene signatures (Data Supplement). As expected, claudin-low tumors were enriched in the diffuse histologic type (Data Supplement). Claudin-low tumors were primarily found in the ACRG EMT subtype (25 of 28), and approximately one half of the ACRG EMT tumors were classified as claudin-low subtype (25 of 44). This finding suggests that the ACRG EMT subtype is also subdivided into claudin-low and non-claudin-low subtypes.

\section{Clinical Characteristics}

Claudin-low tumors were characterized by younger age at diagnosis, with a median age of 58 years in TCGA cohort and 63 years in the ACRG cohort 
Fig 4. Ingenuity pathway analysis (IPA) canonical pathways enriched with differentially expressed genes in claudinlow subtype compared with genomically stable (GS) subtype. Bar graph of the most significantly activated (gold) or inactivated (blue) signaling pathways in claudin-low subtype $(\mathrm{n}=46)$ compared with GS subtype $(\mathrm{n}=41)$.

Significance was

determined using IPA software. A signaling pathway was considered significantly activated (or inhibited) with an overlap $P \leqslant 0.05$ and an IPA activation $z$ score $\geqslant 2.0$ (or $\leqslant-2.0$ ). GDI, guanine nucleotide dissociation inhibitor; NFAT, nuclear factor of activated $\mathrm{T}$ cells.

Fig 5. Prognosis of claudin-low tumors in (A) The Cancer Genome Atlas (TCGA) and (B) the Asian Cancer Research Group (ACRG) data sets. Unadjusted Kaplan-Meier plots show overall survival of gastric cancer by molecular subtype. Significance was determined by log-rank test $(\mathrm{n}=415$ in TCGA cohort; $\mathrm{n}=300$ in the ACRG cohort). CIN chromosomally unstable; EBV, Epstein-Barr virus; GS, genomically stable; MSI, microsatellite unstable.

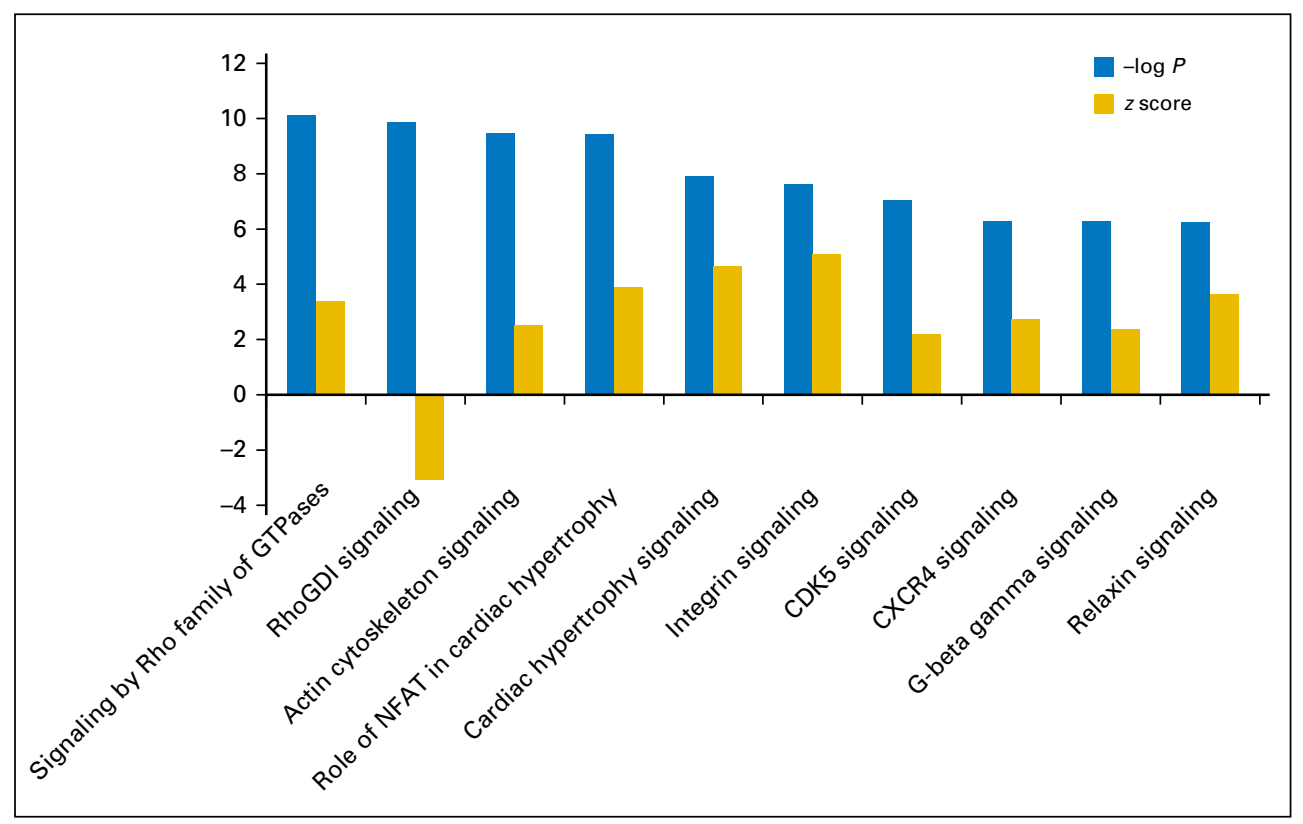

(Data Supplement). To assess prognostic significance of claudin-low subtype, we conducted survival analysis by subtype by using TCGA and ACRG cohorts. Unadjusted Kaplan-Meier survival analysis of TCGA STAD data set showed a trend toward worse prognosis for claudin-low subtype than for the other subtypes (Fig 5A). A similar analysis on the ACRG data set revealed a substantially worse prognosis for claudin-low subtype (Fig 5B). To further compare prognosis for claudin-low subtype with GS subtype, with adjustment for potential confounders (age and pathologic stage), we performed Cox proportional hazards regression modeling by setting GS type as a reference group (Table 1). We observed that claudin-low subtype had significantly worse

overall survival than GS subtype in both TCGA (hazard ratio [HR], 2.10; 95\% CIs, 1.07 to 4.11; $P=.031)$ and the ACRG (HR, 2.32; 95\% CIs, 1.18 to $4.55 ; P=.015)$ cohorts. In addition, we assessed a prognostic value of claudin-low subtype in patients with stage III cancer because it was the largest subgroup with the most equal distribution of subtypes. This analysis also showed that the claudin-low subtype was associated with worse overall survival than the GS subtype in both TCGA (HR, 3.11; 95\% CI, 1.10 to 8.75 ; $P=.032)$ and the ACRG (HR, 3.67; 95\% CI, 1.43 to $9.43 ; P=.007)$ cohorts. Similar HRs from the two independent data sets suggest that claudin-low subtype of gastric cancer has a poor prognosis as a result of its unique biologic features.

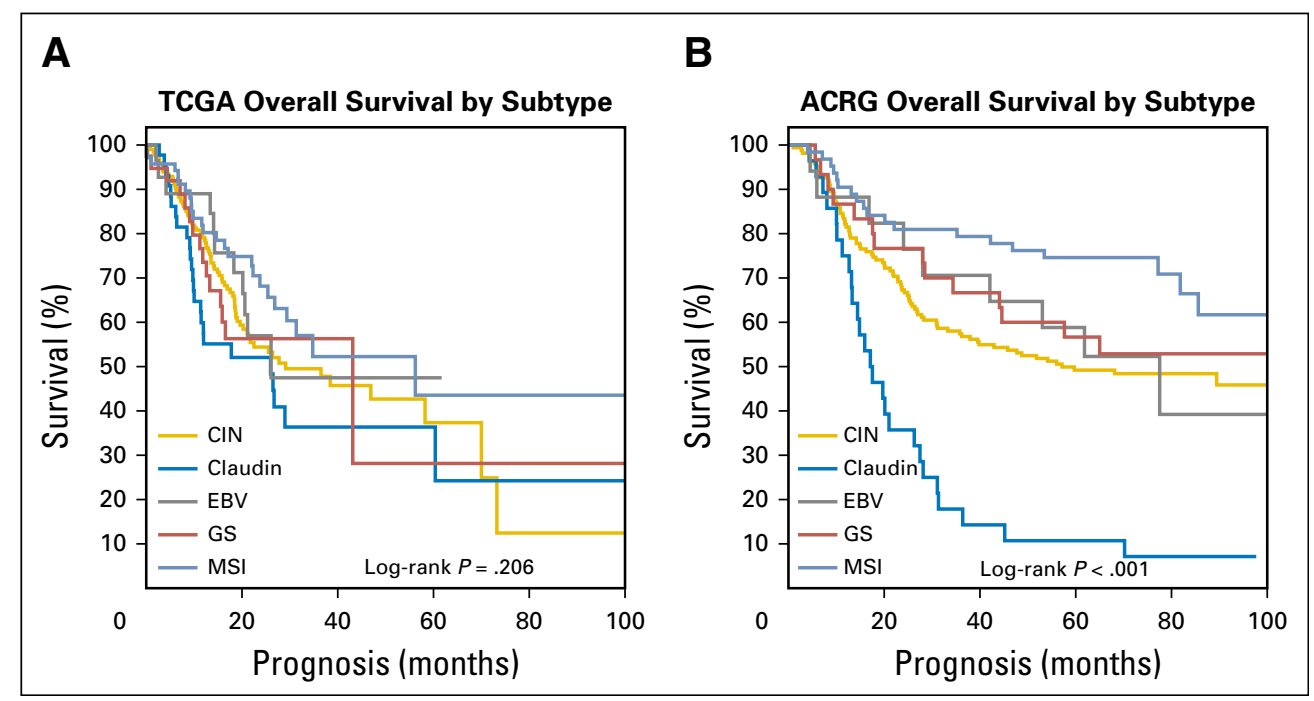




\section{DISCUSSION}

We characterized the claudin-low subtype of gastric cancer. Approximately $80 \%$ of claudin-low tumors were originally classified as TCGA GS subtype. Thus, we especially focused on comparing molecular and clinical features of claudin-low tumors with those of non-claudin-low GS tumors. Claudin-low gastric cancer was defined by low expression levels of tight-junction claudins, high levels of EMT, and enrichment for TIC signatures. In addition, compared with GS subtype, claudin-low subtype had the lower expression of proliferation signature, significant activation in Rho family of GTPases signaling, and enrichment of cell motility and adhesion-associated pathways. Furthermore, the claudin-low subtype conferred significantly worse prognosis than the GS subtype, which had comparable survival to the CIN and EBV subtypes. These observations support the claim that claudin-low subtype is distinct from GS subtype and warrants additional validation in prospective studies.

We report a 24-gene classifier that accurately classifies gastric cancer into claudin-low and nonclaudin-low subtypes. We applied this classifier to the ACRG data set and identified 28 claudin-low tumors that were phenotypically similar to the initially derived claudin-low subtype in the discovery data set. Claudin-low tumors were primarily found in the ACRG EMT subtype (25 of 28). This finding is keeping with the notion that high expression of EMT signatures is one of the key features that define claudin-low tumors but is not a sufficient condition because claudin-low tumors

Table 1. Cox Proportional Hazards Regression Modeling for Overall Survival (TCGA and ACRG cohorts)

\begin{tabular}{lccc}
\multicolumn{1}{c}{ Cohort } & Hazard Ratio & $\mathbf{9 5 \%}$ CI & $\boldsymbol{P}$ \\
\hline TCGA (reference group = GS) & & & \\
\hline Claudin-low & 2.10 & 1.07 to 4.11 & .031 \\
\hline CIN & 1.02 & 0.57 to 1.81 & .961 \\
\hline EBV & 1.01 & 0.44 to 2.28 & .989 \\
\hline MSI & 0.65 & 0.33 to 1.29 & .216 \\
\hline ACRG (reference group = GS) & & & \\
\hline Claudin-low & 2.32 & 1.18 to 4.55 & .015 \\
\hline CIN & 1.05 & 0.58 to 1.90 & .879 \\
\hline EBV & 0.82 & 0.35 to 1.93 & .643 \\
\hline MSI & 0.60 & 0.29 to 1.24 & .169
\end{tabular}

NOTE. Adjusted for age and pathologic stage ( $\mathrm{n}=415$ in TCGA cohort; $\mathrm{n}=300$ in the ACRG cohort). Abbreviations: ACRG, Asian Cancer Research Group; CIN, chromosomally unstable; EBV, EpsteinBarr virus; GS, genomically stable; MSI, microsatellite unstable; TCGA, The Cancer Genome Atlas. are also characterized by low expression of claudins and enrichment for TIC signatures. As a result, only approximately one half of the ACRG EMT tumors were classified as claudin-low subtype (25 of 44), which suggests that the claudinlow tumors are a subpopulation of the ACRG EMT subtype.

Poor prognosis in patients with gastric claudinlow tumors likely is related to EMT and TIC properties. The combined expression of EMT and cancer stem-cell makers was reported to be associated with aggressive clinical features, such as vascular invasion and metastasis, and prognostic of poorer disease-free and overall survival in a study of 276 patients with gastric cancer. ${ }^{25}$ Moreover, EMT and/or stem-cell-like biologic processes have been linked to resistance to chemotherapy and radiation. ${ }^{26-28}$ The mechanisms of therapeutic resistance include relative quiescence, expression of ATP-binding cassette transporters, an active DNA-repair capacity, and a resistance to apoptosis. ${ }^{29}$ Recent work by Yoon et $\mathrm{al}^{22}$ demonstrated a role of RhoA, a member of the Rho family of GTPases, in gastric cancer stem-like cells (CSCs) for the maintenance of EMT phenotypes and chemotherapy resistance. Inhibition of RhoA in diffuse gastric CSCs blocked spheroid formation, migration, and invasion. Furthermore, RhoA pathway inhibition reversed chemoresistance in diffuse gastric CSCs resistant to fluorouracil and cisplatin. Of note, our IPA revealed that, compared with GS subtype, the most significantly activated and inactivated pathway in claudin-low subtype were Rho family of GTPases signaling and RhoGDI signaling, respectively. GDIs are regulators of Rho GTPases and prevent activation of GTPases by acting as molecular chaperones. ${ }^{30}$ All things considered, dysregulated Rho GTPase signaling appears to play a key role in the biology of gastric claudinlow tumors and contributes to the poor prognosis. Given the chemoresistance in gastric CSCs, a specific CSC-targeted therapy must be used in conjunction with conventional chemotherapy to improve the outcome of claudinlow tumors. To this end, Yoon et $\mathrm{al}^{22}$ tested a Rho-associated protein kinase inhibitor, fasudil, because it is a major downstream effector of RhoA. They showed that fasudil worked similarly to direct RhoA inhibition with RhoA short hairpin RNA in reversing the CSC phenotypes and chemoresistance. In addition, fasudil worked synergistically with cisplatin chemotherapy in xenograft models. Given that fasudil 
is already approved in Japan for use in cerebral vasospasm secondary to subarachnoid hemorrhage, ${ }^{31}$ future studies that explore the combination of fasudil and chemotherapy for treatment of gastric claudin-low tumors will be of great interest.

DOI: https://doi.org/10.1200/PO.17.00047

Published online on ascopubs.org/journal/po on June 19, 2017.

\section{AUTHOR CONTRIBUTIONS}

Conception and design: Tomohiro F. Nishijima,

Michael S. Lee, Benjamin G. Vincent

Financial support: Benjamin G. Vincent

Administrative support: Benjamin G. Vincent

Collection and assembly of data: Tomohiro F. Nishijima,

Jordan Kardos, Shengiie Chai

Data analysis and interpretation: Tomohiro F. Nishijima, Shengjie Chai, Christof C. Smith, Dante S. Bortone, Sara R. Selitsky, Hanna K. Sanoff, Michael S. Lee, Benjamin G. Vincent

Manuscript writing: All authors

Final approval of manuscript: Tomohiro F. Nishijima, Shengjie Chai, Christof C. Smith, Dante S. Bortone, Sara R. Selitsky, Hanna K. Sanoff, Michael S. Lee, Benjamin G. Vincent

Accountable for all aspects of the work: All authors

\section{AUTHORS' DISCLOSURES OF}

POTENTIAL CONFLICTS OF INTEREST

Molecular and Clinical Characterization of a ClaudinLow Subtype of Gastric Cancer

The following represents disclosure information provided by authors of this manuscript. All relationships are considered compensated. Relationships are self-held unless noted. I = Immediate Family Member, Inst = My Institution. Relationships may not relate to the subject matter of this manuscript. For more information about ASCO's conflict of interest policy, please refer to www.asco.org/rwc or po.ascopubs.org/site/ifc.
Tomohiro F. Nishijima

No relationship to disclose

Jordan Kardos

No relationship to disclose

Shengjie Chai

No relationship to disclose

Christof C. Smith

No relationship to disclose

Dante S. Bortone

Employment: Johnson \& Johnson (I)

Patents, Royalties, Other Intellectual Property: patent submitted for fluorescence and magnetic resonance-detectable retrograde neural tracers

Sara R. Selitsky

No relationship to disclose

Joel S. Parker

No relationship to disclose

Hanna K. Sanoff

Research Funding: Bayer AG (Inst), Novartis (Inst), Merck (Inst), Precision Biologics (Inst), Immunomedics (Inst)

Michael S. Lee

No relationship to disclose

Benjamin G. Vincent

No relationship to disclose

\section{Affiliations}

All authors: University of North Carolina at Chapel Hill, Chapel Hill, NC.

\section{REFERENCES}

1. Prat A, Parker JS, Karginova O, et al: Phenotypic and molecular characterization of the claudin-low intrinsic subtype of breast cancer. Breast Cancer Res 12:R68, 2010

2. Kardos J, Chai S, Mose LE, et al: Claudin-low bladder tumors are immune infiltrated and actively immune suppressed. JCI Insight 1:e85902, 2016

3. Sabatier R, Finetti P, Guille A, et al: Claudin-low breast cancers: Clinical, pathological, molecular and prognostic characterization. Mol Cancer 13:228, 2014

4. Chia NY, Tan P: Molecular classification of gastric cancer. Ann Oncol 27:763-769, 2016

5. Cristescu R, Lee J, Nebozhyn M, et al: Molecular analysis of gastric cancer identifies subtypes associated with distinct clinical outcomes. Nat Med 21:449-456, 2015

6. Lei Z, Tan IB, Das K, et al: Identification of molecular subtypes of gastric cancer with different responses to PI3-kinase inhibitors and 5-fluorouracil. Gastroenterology 145:554-565, 2013

7. Cancer Genome Atlas Research Network: Comprehensive molecular characterization of gastric adenocarcinoma. Nature 513:202-209, 2014

8. Broad Institute: Firehose Broad GDAC. http://gdac.broadinstitute.org

9. Herschkowitz JI, Simin K, Weigman VJ, et al: Identification of conserved gene expression features between murine mammary carcinoma models and human breast tumors. Genome Biol 8:R76, 2007 
10. Tan TZ, Miow QH, Miki Y, et al: Epithelial-mesenchymal transition spectrum quantification and its efficacy in deciphering survival and drug responses of cancer patients. EMBO Mol Med 6:1279-1293, 2014

11. Hippo Y, Taniguchi H, Tsutsumi S, et al: Global gene expression analysis of gastric cancer by oligonucleotide microarrays. Cancer Res 62:233-240, 2002

12. Iglesia MD, Vincent BG, Parker JS, et al: Prognostic B-cell signatures using mRNA-seq in patients with subtypespecific breast and ovarian cancer. Clin Cancer Res 20:3818-3829, 2014

13. Strong MJ, Xu G, Coco J, et al: Differences in gastric carcinoma microenvironment stratify according to EBV infection intensity: Implications for possible immune adjuvant therapy. PLoS Pathog 9:e1003341, 2013

14. Fridman WH, Galon J, Dieu-Nosjean MC, et al: Immune infiltration in human cancer: Prognostic significance and disease control. Curr Top Microbiol Immunol 344:1-24, 2011

15. Iglesia MD, Parker JS, Hoadley KA, et al: Genomic analysis of immune cell infiltrates across 11 tumor types. J Natl Cancer Inst 108:djw144, 2016

16. Sharma P, Shen Y, Wen S, et al: CD8 tumor-infiltrating lymphocytes are predictive of survival in muscle-invasive urothelial carcinoma. Proc Natl Acad Sci U S A 104:3967-3972, 2007

17. Mahmoud SM, Paish EC, Powe DG, et al: Tumor-infiltrating CD8 lymphocytes predict clinical outcome in breast cancer. J Clin Oncol 29:1949-1955, 2011

18. Gooden MJ, de Bock GH, Leffers N, et al: The prognostic influence of tumour-infiltrating lymphocytes in cancer: A systematic review with meta-analysis. Br J Cancer 105:93-103, 2011

19. Lee HE, Chae SW, Lee YJ, et al: Prognostic implications of type and density of tumour-infiltrating lymphocytes in gastric cancer. Br J Cancer 99:1704-1711, 2008

20. Shen Z, Zhou S, Wang Y, et al: Higher intratumoral infiltrated Foxp3+ Treg numbers and Foxp3+/CD8+ ratio are associated with adverse prognosis in resectable gastric cancer. J Cancer Res Clin Oncol 136:1585-1595, 2010

21. Liu K, Yang K, Wu B, et al: Tumor-infiltrating immune cells are associated with prognosis of gastric cancer. Medicine (Baltimore) 94:e1631, 2015

22. Yoon C, Cho SJ, Aksoy BA, et al: Chemotherapy resistance in diffuse-type gastric adenocarcinoma is mediated by RhoA activation in cancer stem-like cells. Clin Cancer Res 22:971-983, 2016

23. Brungs D, Aghmesheh M, Vine KL, et al: Gastric cancer stem cells: Evidence, potential markers, and clinical implications. J Gastroenterol 51:313-326, 2016

24. Chia NY, Deng N, Das K, et al: Regulatory crosstalk between lineage-survival oncogenes KLF5, GATA4 and GATA6 cooperatively promotes gastric cancer development. Gut 64:707-719, 2015

25. Ryu HS, Park DJ, Kim HH, et al: Combination of epithelial-mesenchymal transition and cancer stem cell-like phenotypes has independent prognostic value in gastric cancer. Hum Pathol 43:520-528, 2012

26. Takaishi S, Okumura T, Tu S, et al: Identification of gastric cancer stem cells using the cell surface marker CD44. Stem Cells 27:1006-1020, 2009

27. Yoon C, Park DJ, Schmidt B, et al: CD44 expression denotes a subpopulation of gastric cancer cells in which hedgehog signaling promotes chemotherapy resistance. Clin Cancer Res 20:3974-3988, 2014

28. Xu ZY, Tang JN, Xie HX, et al: 5-Fluorouracil chemotherapy of gastric cancer generates residual cells with properties of cancer stem cells. Int J Biol Sci 11:284-294, 2015

29. Dean M, Fojo T, Bates S: Tumour stem cells and drug resistance. Nat Rev Cancer 5:275-284, 2005

30. Porter AP, Papaioannou A, Malliri A: Deregulation of Rho GTPases in cancer. Small GTPases 7:123-138, 2016

31. Hirooka Y, Shimokawa H: Therapeutic potential of Rho-kinase inhibitors in cardiovascular diseases. Am J Cardiovasc Drugs 5:31-39, 2005 